\title{
Minimal Laparotomy Management of a Giant Asymptomatic Ovarian Teratoma in a Woman of Reproductive Age: A Case Report
}

\author{
Amal AL Mulla ${ }^{1 *}$, Mohamed EL Houssainy ${ }^{2}$, Munaf Desai ${ }^{3}$ and Shahid Siddiqui ${ }^{4}$ \\ ${ }^{1}$ Consultant OBSGYN \& Reproductive Medicine, Saudi German Hospital, United Arab Emirates \\ ${ }^{2}$ Specialist Radiologist, Saudi German Hospital, United Arab Emirates \\ ${ }^{3}$ Specialist Histopathologist \& Laboratory Director, Saudi German Hospital, United Arab Emirates \\ ${ }^{4}$ Specialist Anesthesiologist, Saudi German Hospital, United Arab Emirates
}

*Corresponding author: Amal AL Mulla, Consultant OBSGYN \& Reproductive Medicine, Saudi German Hospital, Dubai, United Arab Emirates

Received Date: June 25, 2020

Published Date: July 02, 2020

\begin{abstract}
Background: The majority of giant teratomas are symptomatic. The preoperative evaluation for possible malignancy is challengeable, as is the surgical approach. A consensus has not yet been drawn regarding the optimal treatment approach in cases of exceptionally large teratomas.

Case presentation: We report a rare case of minimal laparotomy management of a giant ovarian cystic teratoma in a 32-year-old asymptomatic multiparous woman. The patient was diagnosed while performing a fitness-to-work checkup where the physician noted a suspicious abdominal swelling. Subsequently, she was referred to us for further evaluation. Her CA125 level was raised, and an abdominal contrast computed tomography revealed a huge teratoma. After appropriate counselling, laparotomy and adenectomy were performed. Histopathological examination diagnosed a mature cystic teratoma with no malignant transformation. The patient had an uneventful recovery and was discharged on the third post-operative day.

Conclusions: We conclude that dermoid cysts can reach enormous sizes in the absence of serious symptoms or evidence of malignancy, although the size is a well-known risk factor for malignant transformation.
\end{abstract}

Keywords: Giant teratoma; Pelvic mass; Laparotomy; Computed tomography

Abbreviations: AFP: $\alpha$-Fetoprotein; CEA: Carcino Embryonic Antigen; CT: Computed Tomography; SCC Ag: Squamous Cell Carcinoma Antigen

\section{Background}

Ovarian tumors can originate from any of its different layers. The three major histological types are: epithelial tumors, sex cord stromal cell tumors, and germ cell tumors, which constitute approximately $25 \%$ of all ovarian tumors (Figure 1) [1].

Teratomas are slow-growing tumors, composed histologically of diverse tissues, such as bone, skin, sebaceous glands, etc., owing to its cell of origin. Pathologically, teratomas are classified into benign mature, malignant immature, and mono-dermal types that are composed of one type of tissue [2]. In 10-17\% of cases, both ovaries are involved [3].

Ovarian teratomas rarely exceeds the diameter of $15 \mathrm{~cm}$. Teratomas that exceed this size have an increased likelihood of 
malignant transformation. Other risk factors include elevated tumor markers and advanced age [4]. Giant teratomas usually present with complications, including torsion, pressure symptoms, and rupture. Here, we report a rare case of an asymptomatic giant teratoma in a 32-year-old multiparous woman. The CARE guidelines were followed for this case report [5].

\section{Case Presentation}

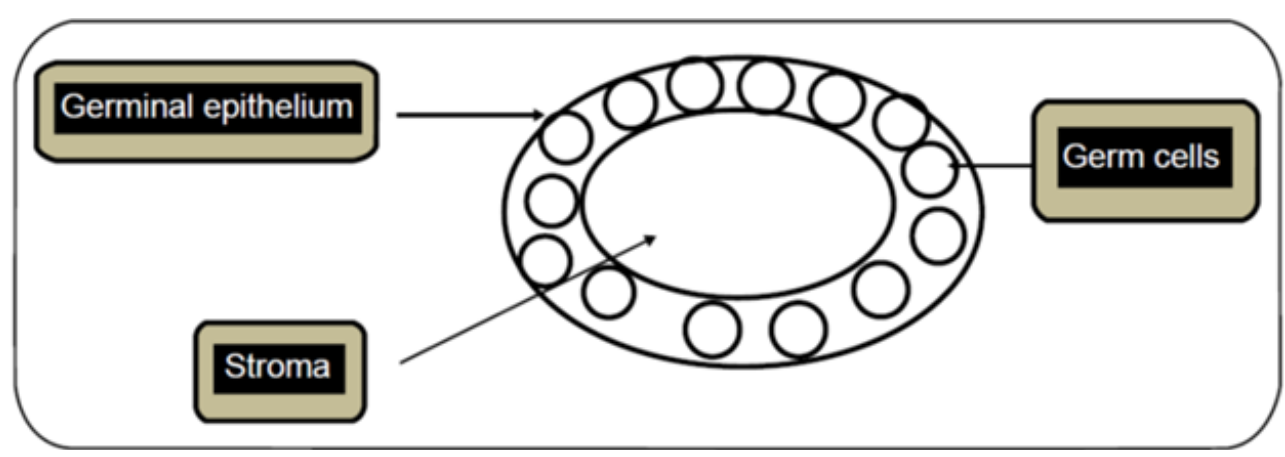

Figure 1: Simplified diagram illustrating different layers of the ovary.

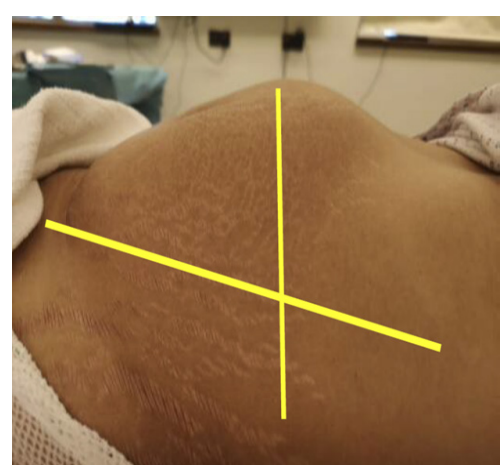

Figure 2: A diffuse swelling occupying the entire abdominal and pelvic cavity.

A 32-year-old multiparous woman was referred for further evaluation of a suspicious abdominal mass. During a discussion of the patient's medical history, the patient admitted to the existence of this mass for more than one year in association with vague, intermittent abdominal pain. She reported no changes in her menstrual cycle or bowel habits. There was no other significant medical or family history. On inspection of the abdomen, a clear bulge was evident approximately $20 \times 30 \mathrm{~cm}$ in size in the periumbilical region. It was soft, and no area of tenderness could be detected (Figure 2).

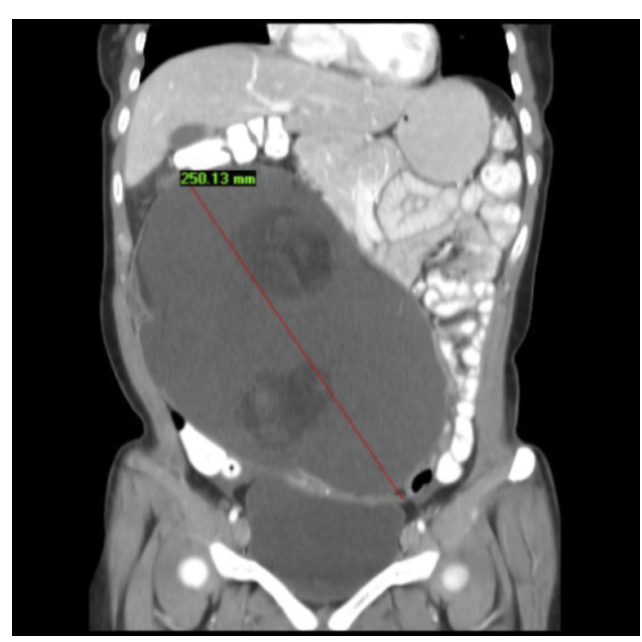

Figure 3: Coronal section of the abdominal computed tomography scan. 
Due to its huge size, an extensive workup was performed to assess the possible risks of malignancy. The levels of serum tumor markers $\alpha$-fetoprotein (AFP), carcinoembryonic antigen (CEA), and $\beta$-human chorionic gonadotropin, were normal. However, the patient's CA125 level was abnormally high at $50 \mathrm{U} / \mathrm{ml}$ (normal range, $<35 \mathrm{U} / \mathrm{ml}$ ). Abdominal contrast computed tomography (CT) revealed a $25 \times 20 \times 14 \mathrm{~cm}$ pelviabdominal cystic multilocular mass with fluid/fat density and internal nodules of fat and calcific teeth (Rokitansky nodules) suggestive of a dermoid cyst, originating from the left ovary. Displacement of the surrounding bowel loops and diffuse anterior abdominal wall contour bulge were also noted (Figure 3). There was no evidence of enlarged lymph nodes, ascites, or tumor deposits in the abdomen or pelvis.

Other blood test findings were within the normal ranges, and liquid base cytology was negative for malignant cells. The radiological and laboratory findings were discussed in detail with the patient, and the possible risks of malignancy were highlighted. Written informed consent for surgery was obtained.

A mini-midline laparotomy was performed. A huge mass was seen originating from the left ovary, extending to the liver, with an approximate size of $20 \times 40 \times 20 \mathrm{~cm}$. The fallopian tube was stretched out by the mass. The cyst was aspirated completely without spillage in the peritoneal cavity. A total $5 \mathrm{~L}$ of thick, yellowish fluid was aspirated. No ascites or organ infiltrate was noted. Complete adenectomy was performed due to the absence of a cleavage line between the cyst and the ovary; the ovarian tissue was completely destroyed by the mass (Figure 4a). However, the contralateral tube and uterus were normal. The patient had an uneventful recovery and was discharged on the third post-operative day.
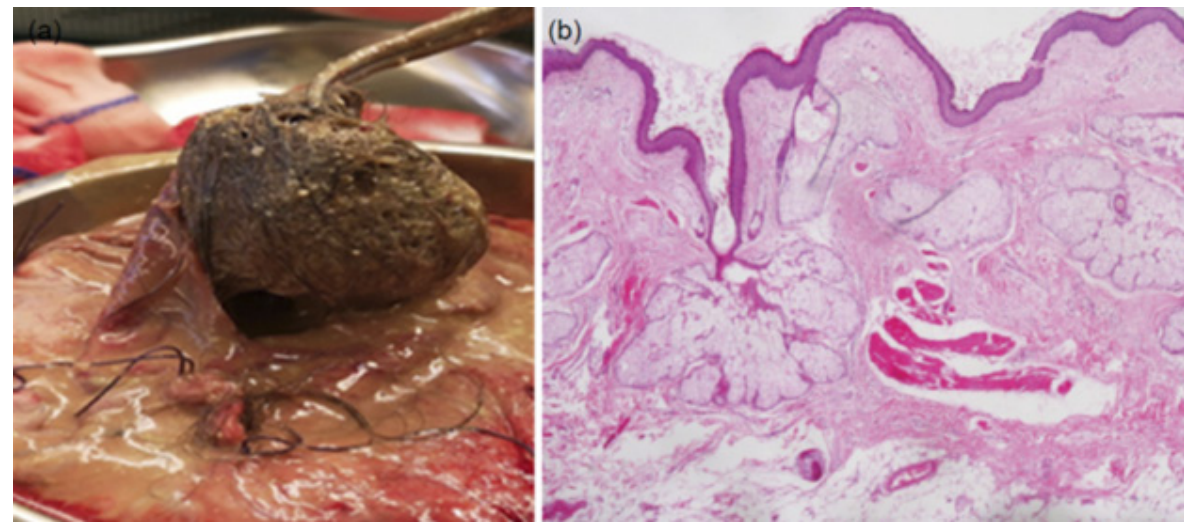

Figure 4: (a) Intra-operative macroscopic findings: multicyclic tissues filled with creamy material. (b) Cross-sectional analysis revealed skin with appendages (H \& E, 10x).

Histopathological assessment revealed a mature cystic teratoma composed of skin with appendages (Figure $4 \mathrm{~b}$ ), bone with marrow in addition to muscle tissues, nerve bundles, and glial tissues. Scarce ovarian tissue was included in a part of the cyst's wall. No foci of malignant change could be detected. These findings were communicated to the patient post-operatively. A remarkable reduction in her weight by approximately $5 \mathrm{~kg}$ was noted during a visit to the clinic 10 days post-operation. In addition, she reported feeling light with an improved level of physical activity.

\section{Discussion}

Although teratomas are common, reaching an abnormally huge size that exceeds $15 \mathrm{~cm}$ in diameter is rare. In a retrospective study conducted by Ayhan, et al. 501 operative patients' records between 1964 and 1998 at Hacettepe University Hospital were examined, and the mean tumor diameter was $7.0 \pm 4.5 \mathrm{~cm}[6]$.

Teratomas are encountered in women of reproductive age, with rare cases having been reported at extreme ages. Asymptomatic giant teratomas are extremely rare because, since they occupy most of the abdominal-pelvic cavity, they will exert pressure on the surrounding structures or torsion, causing pressure/pain symptoms. Furthermore, over-stretching of the capsule with the collected material may amount to capsular rupture, chemical peritonitis, and dissemination of malignant cells, if present [7].

Although malignant transformation is rare, approximately 1-2\% of teratoma cases become malignant [8]. Diagnosing malignancy in a giant cystic teratoma preoperatively is not easy. The role of elevated values of serum tumor markers is not fully understood; while some have reported that SCC Ag (squamous cell carcinoma antigen) is a useful marker to indicate squamous cell carcinoma in mature cystic teratomas, others found that CA125 and CA19-9 can be elevated in benign teratomas [3].

Ultrasonography has a very high positive predictive value in diagnosing ovarian teratomas, but it is limited in assessing the coexitance of metastasis. Therefore, additional CT scans or magnetic resonance imaging are required for optimum preoperative assessment of giant teratomas. Fat attenuation within a cyst with or without calcification is pathognomonic for mature cystic teratoma $[9,10]$. 
Surgical excision is fundamental to obtain a histopathological diagnosis and to detect the presence of immature elements. Both laparoscopic and open approach have been described to excise giant teratomas. However, the high possibility of spillage of teratoma contents is a procedure-related risk, which can be noted in up to $100 \%$ of laparoscopic surgery cases and $13 \%$ of laparotomy cases [11].

Spillage of the contents is associated with a substantial risk of chemical peritonitis and dissemination of malignant cells. The incidence rate of chemical peritonitis owing to granulomatous peritonitis is approximately $8 \%$ of cases [12]. Furthermore, prelaparoscopic decompression might be impractical in cases of solid teratomas. Further, using the laparoscopic approach for masses $>10 \mathrm{~cm}$ in size is debatable [13]. A retrospective study of 306 cases reported that laparoscopic surgery was performed in $57.8 \%(n=177)$ of patients, and cyst rupture occurred in $19(6.2 \%)$ patients [8]. Shalev and Peleg recommended laparoscopic surgery as routine treatment for ovarian teratomas as large as $15 \mathrm{~cm}$ in diameter [14]. Nevertheless, this is not recommended by many investigators, especially if malignancy is suspected [15]. Tsalkidou et al. performed an exploratory laparotomy in a 26-year-old woman with a huge benign cystic teratoma measuring $14 \times 20 \times 18 \mathrm{~cm}$ who presented with progressive abdominal distension and discomfort five years after giving birth [15]. Further, Barghi, et al. performed a successful percutaneous drainage of $18.5 \mathrm{~L}$ of fluid followed by a mini-laparotomy for a $38 \times 32 \times 23 \mathrm{~cm}$ mucinous ovarian cystadenoma in association with a mature cystic teratoma in a 26-year-old woman who had progressive abdominal distension over several years [16].

\section{Acknowledgement}

None.

\section{Conflict of Interest}

Authors declare no conflict of interest.

\section{References}

1. Chen VW, Ruiz B, Killeen JL, Coté TR, Wu XC, et al. (2003) Pathology and classification of ovarian tumors. Cancer $97(10 \mathrm{Suppl}): 2631-2642$
2. Goudeli C, Varytimiadi A, Koufopoulos N, Syrios J, Terzakis E (2016) An ovarian mature cystic teratoma evolving in squamous cell carcinoma: A case report and review of the literature. Gynecol Oncol Rep 19: 27-30.

3. Hackethal A, Brueggmann D, Bohlmann MK, Franke FE, Tinneberg HR, et al. (2008) Squamous-cell carcinoma in mature cystic teratoma of the ovary: systematic review and analysis of published data. Lancet Oncol 9(12): 1173-1180.

4. Park CH, Jung MH, Ji YI (2015) Risk factors for malignant transformation of mature cystic teratoma. Obstet Gynecol Sci 58(6): 475-80.

5. Gagnier JJ, Kienle G, Altman DG, Moher D, Sox H, et al. (2014) The CARE guidelines: consensus-based clinical case report guideline development. J Clin Epidemiol 67(1): 46-51.

6. Ayhan A, Bukulmez 0, Genc C, Karamursel BS, Ayhan A (2000) Mature cystic teratomas of the ovary: case series from one institution over 34 years. Eur J Obstet Gynecol Reprod Biol 88(2): 153-157.

7. Ye LY, Wang JJ, Liu DR, Ding GP, Cao LP (2012) Management of giant ovarian teratoma: A case series and review of the literature. Oncol Lett 4(4): 672-676.

8. Avcı S, Selcukbiricik F, Bilici A, Özkan G, Özağarı AA, et al. (2012) Squamous cell carcinoma arising in a mature cystic teratoma. Case Rep Obstet Gynecol 2012: 1-3.

9. Bernot JM, Haeusler KA, Lisanti CJ, Brady RO, Ritchie BL (2017) Mature cystic teratoma: AIRP best cases in radiologic-pathologic correlation. Radiographics 37(5): 1401-1407.

10. Saba L, Guerriero S, Sulcis R, Virgilio B, Melis G, Mallarini G (2009) Mature and immature ovarian teratomas: CT, US and MR imaging characteristics. Eur J Radiol 72(3): 454-463.

11. Kondo W, Bourdel N, Cotte B, Tran X, Botchorishvili R, et al. (2010) Does prevention of intraperitoneal spillage when removing a dermoid cyst prevent granulomatous peritonitis? BJOG 117(8): 1027-1030.

12. Comerci Jr JT, Licciardi F, Bergh PA (1994) Mature cystic teratoma: a clinicopathologic evaluation of 517 cases and review of the literature. Obstet Gynecol 84(1): 22-28.

13. Einenkel J, Alexander H, Schotte D (2006) Giant ovarian cysts: Is a preand intraoperative drainage an advisable procedure? Int J Gynecol Cancer 16: 2039-2043.

14. Shalev E, Peleg D (1993) Laparoscopic treatment of adnexal torsion. Surg Gynecol Obstet 176: 448-450.

15. Tsalkidou EG, Memet IH, Chasan GM (2016) Ovarian cystectomy for huge mature cystic teratoma developed in less than five years: a case report. Gynecol Obstet Res Open J 2: 99-101.

16. Barghi A, Wu M, Nguyen V (2018) Minimally invasive drainage of a giant ovarian mucinous cystadenoma associated with a mature cystic teratoma. J Surg Case Rep 18(11). 